Document downloaded from:

http://hdl.handle.net/10251/115616

This paper must be cited as:

Rubio Michavila, C.; Uris Martínez, A.; Minin, IV.; Minin, OV.; Tarrazó-Serrano, D. (2019). Enhancement of pupil-masked wavelength-scale gas-filled flat acoustic lens based on anomaly apodization effect. Physics Letters A. 383:396-399.

doi:10.1016/j.physleta.2018.11.014

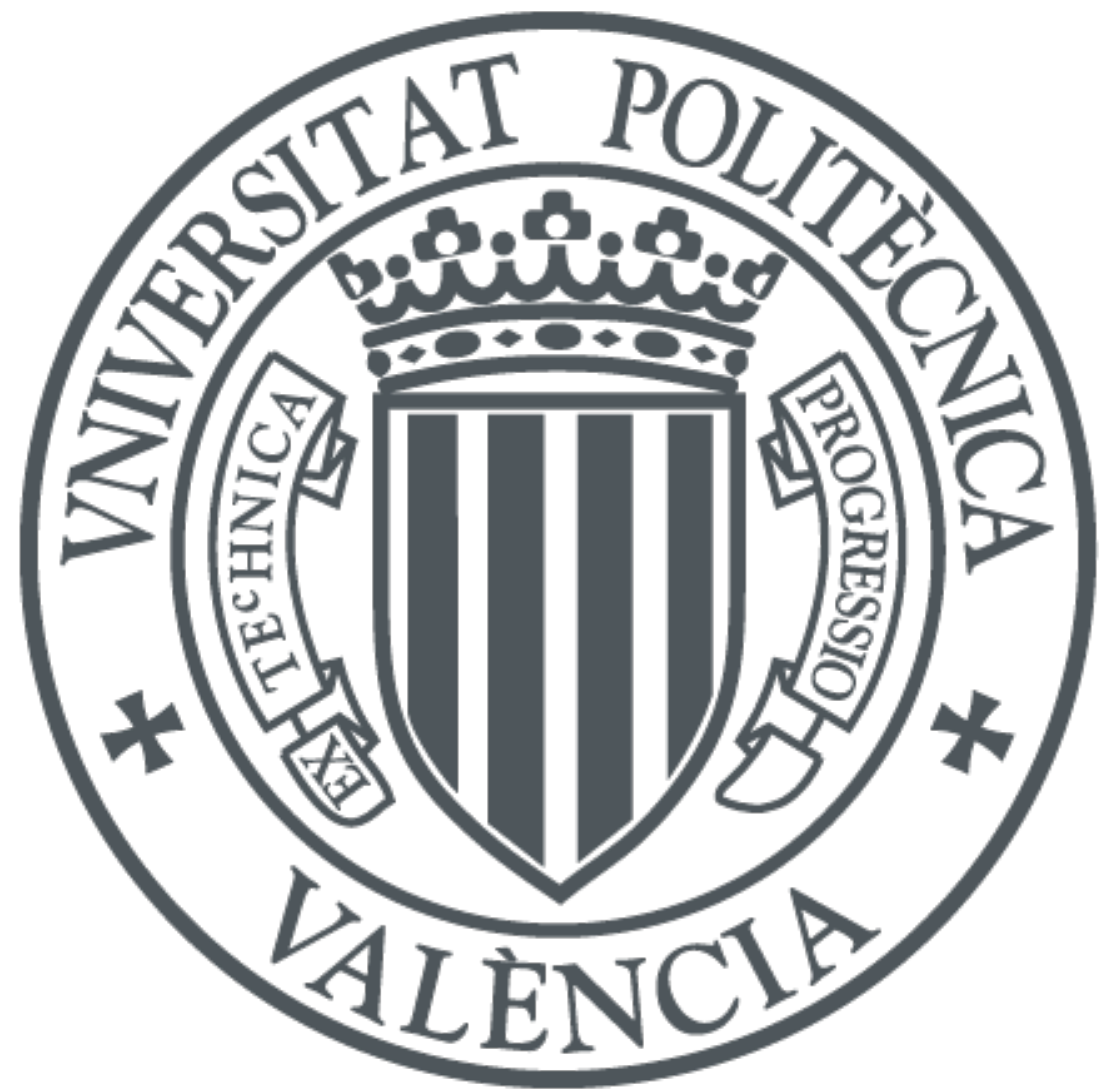

The final publication is available at

https://doi.org/10.1016/j.physleta.2018.11.014

Copyright Elsevier

Additional Information 


\title{
ENHANCEMENT OF PUPIL-MASKED WAVELENGTH-SCALE GAS-FILLED FLAT ACOUSTIC LENS BASED ON ANOMALY APODIZATION EFFECT
}

\section{Constanza Rubio ${ }^{1, *}$, Daniel Tarrazó-Serrano ${ }^{1}$, Oleg V. Minin ${ }^{2}$, Antonio Uris ${ }^{1}$, Igor V.} $\operatorname{Minin}^{3, *}$.

*Corresponding Author: E-mail: crubiom@,fis.upv.es; prof.minin@gmail.com

${ }^{1}$ Centro de Tecnologías Físicas: Acústica, Materiales y Astrofísica, Universitat Politècnica de València, Camino de Vera s/n, 46022 Valencia, Spain.

2 Tomsk State University, 30 Lenin Avenue, Tomsk, 634050, Russia

${ }^{3}$ Tomsk Politechnical University, 36 Lenin Avenue, Tomsk, 634050, Russia

\begin{abstract}
In this letter, the improvement in focus by the use of a pupil mask produced in an acoustic mesoscale cuboid particle filled with $\mathrm{CO}_{2}$ is reported. Thereby, the result shows that the pupil mask increases the sound intensity and also increases the resolution (or a reduction of the full width at half maximum, FWHM) in focus compared to the non-masked one. These results are important because they confirm the effect of abnormal amplitude apodization for a onewavelength dimension acoustic lens and demonstrate that it is possible to improve sound focusing of a cuboid gas-filled lens with one wavelength dimension. This is the smallest size of an acoustic lens ever considered in this type of literature, with side dimensions of the cube equal to one wavelength and a diameter to focus ratio of 2.5 , the sound amplification in focus is 5.4 $\mathrm{dB}$ at $4125 \mathrm{~Hz}$, with the resolution near to the diffraction limit.
\end{abstract}

Keywords: Sound focusing, gas-filled lens, cuboid particle, apodization, diffraction

\section{Introduction}

Lenses are devices that guide waves to follow a certain path. One of the most important lens design aspects is transmission efficiency, especially in the case of acoustic lenses due to a lack of materials that simultaneously have a high refractive index and a low contrast of impedances 
with the host medium. In acoustics research, the study of acoustic lenses is an important issue that has attracted interest for a long time and currently continues to stimulate a vast amount of research.

Although the first acoustic lens was a gas-filled lens that used carbon dioxide and was created in 1852 by Sondhauss [1], there are only a few published works on the idea of using a gas-filled lens to focus sound. The existing published works use the lower gas velocity inside the lens and the curvature of the surface to produce the focalization and that is why they use balloon gasfilled lens $[2,3]$. Nevertheless, different lens designs with different mechanisms to produce wave focalization have been proposed. For example, thin Fresnel diffractive lenses have been designed and fabricated for focusing underwater ultrasound [4], phononic crystal lenses $[5,6]$, gradient acoustic lenses designed by using space-coiling structures [7-10], lenses that exploit Fabry-Perot resonances to increase the transmitted energy [11, 12], a metamaterial-based gasfilled layers lens, which, however, requires the precision manufacture of gas layers and the use of at least two different gases (argon and xenon) [13], metamaterial-based lenses in reflection mode [14-15], etc.

On the other hand, in recent years, the study of the phenomenon of the Photonic Jet (PJ) both in transmitting [16] and reflection [17] modes, has received much interest within the scientific community not only for clarifying the physics behind the phenomenon, but also because of its potential applications [16]. When a plane electromagnetic wave illuminates a particle-lens dielectric, a high-intensity focus is produced on the shadow surface of the particle. This highintensity focusing area is called photonic jet. The factors on which the behaviour of the PJ depends are the contrast of the refractive index, and the size and geometry of the particle [16]. But diffraction in common cases leads to some unfavourable effects, such as an increase in the size of a beam during propagation, and a limited minimal beam size after focusing. To overcome these disadvantages, the apodization technique is applied to reduce the divergence of a beam 
and increase the resolution while decreasing the intensity in the focus [18]. However, intensity loss is always unavoidable in the system applied by this method, because the centred pupil mask lowers the amount of the incoming wave front to be admitted in the acoustic lens [19-20]. In the last three years, many optical studies regarding the mask apodization in dielectric particlelenses of different shapes have been carried out [21-25]. It was discovered that pupil-masked cylindrical columns and cuboid particle lenses are not only able to produce photonic jets with a shorter beam waist, but also increase its maximal intensity at certain masking ratios on the receiving surface, by the so called anomaly apodization effect, observed in optical and $\mathrm{THz}$ bands [23-25].

Recently, the ideas initially developed for electromagnetic waves have been transferred to the field of acoustics motivated by the nature of both acoustic and electromagnetic waves. The socalled "acoustojets" phenomenon was reported theoretically for the first time by Minin's [2627] and they demonstrated the existence of an acoustic analogue the of photonic jet phenomenon, i.e., the existence of high acoustic field localization in the shadow area of an 3D particle. The acoustojet phenomenon was experimentally verified in the ultrasonic range for spherical [28] and cylindrical [29] dielectric particle-lenses immersed in water. Recently, we have shown, both numerically and experimentally, for the first time, that an acoustic mesoscale dimensioned flat cuboid-particle filled with $\mathrm{CO}_{2}$ focuses sound just as conventional curved acoustic lenses do [30]. It was also shown that the focusing capabilities of such a lens are close to the diffraction limit and a gas-filled lens has advantages in comparison with a solid particlelens due to a low contrast of impedances with the host medium.

In this letter, we report the mask apodization in a mesoscale flat cuboid particle acoustic lens with a side length equal to the incident wavelength $(\lambda)$ and filled with $\mathrm{CO}_{2}$. The focusing produced by a pupil masked flat cuboid is numerically simulated. The modelling result shows 
that the flat cuboid particle acoustic lens with an optimal pupil mask has a higher enhancement of sound intensity and a smaller beam waist (FWHM) compared to the non-masked one.

\section{Numerical results and discussion}

The analysed structure is a cuboid of side length $L$ with a pupil mask located on the face where the plane wave falls upon. The pupil mask is a square slab of side $p s$, as shown in Figure 1. In order to mathematically implement the characteristics of the system and to physically analyse the problem involved, a Finite Element Method (FEM) has been used by means of the Acoustic Model of COMSOL Multiphysics Modeling(C). Given the high computational cost required by this model, a symmetric boundary located at the centre of the model has been defined. This condition emulates a mirror-plane that accomplishes $\mathrm{s}=-\mathrm{s}$, where $\mathrm{s}$ is any acoustic magnitude without being a proper characteristic of the medium where the acoustic wave propagates. This fact allows us, to simulate the half of the domain, therefore requiring a smaller number of partial differential equations that are called degrees of freedom (DoF) in FEM, to diminish the burden of 3D-models. The domain is formed by a cube of $\mathrm{CO}_{2}$ of $80 \times 80 \times 80 \mathrm{~mm}^{3}$ (or $\lambda \times \lambda \times \lambda$ at $4125 \mathrm{~Hz}$ ) dimensions placed in a larger air cylinder of radius $4 \lambda$ and length $6 \lambda$. Perfectly Matched Layers (PML) [31], a good alternative to simulate the Sommerfeld radiation conditions in the numerical resolution of scattering problems, surrounds this domain. To obtain a plane wave, a Background Pressure Field (BPF) condition is applied to the entire domain. The $\mathrm{CO}_{2}$ was modelled with a sound speed of $\mathrm{c}_{\mathrm{CO} 2}=260 \mathrm{~m} / \mathrm{s}$ and a density equal to $\rho \mathrm{CO}_{2}=1.977 \mathrm{~kg} / \mathrm{m}^{3}$, whereas the values used to model air were $c_{a i r}=343 \mathrm{~m} / \mathrm{s}$ and $\rho_{\text {air }}=1.21 \mathrm{~kg} / \mathrm{m}^{3}$. The mesh type was set to Free Tetrahedral, with a maximum element size of $\lambda / 8$ and a minimum element size of $\lambda / 10$. The area around the mask was meshed with a much smaller mesh size to avoid numerical dispersion and to minimize simulation time as well as to save memory. 
The phenomenon of the focalization of a cuboid gas-filled lens is influenced by its size. A minimum size is required for the wave front inside the cube to convert from convex to concave at the shadow side of the lens $[24,25,30]$. In a cuboid with side length $\lambda$, the distance is not sufficient for the wave front to reach an optimal concavity. Figure 2a shows the normalized sound intensity distributions, $\mathrm{I} / \mathrm{I}_{\text {incident, }}$ for the $\mathrm{CO}_{2}$ filled cuboid lens. An enhancement of 3 , can be observed, which is much smaller than that of a cuboid with dimensions $2 \lambda[30]$. However, when a square pupil-mask of $5 \mathrm{~mm}$ side $(0.0625 \lambda)$ of a material that is opaque to the sound (in our case steel) is placed in the centre of the cuboid face encountering the plane wave, an increase in the enhancement of around $10 \%$ is observed (Figure $2 \mathrm{~b}$ ). The sound amplification (SA) in focus is defined as $\mathrm{SA}(\mathrm{dB})=10 \cdot \log (\mathrm{I}(\mathrm{x}, \mathrm{y}) / \operatorname{Inncident}(\mathrm{x}, \mathrm{y}))$ and a $\mathrm{SA}$ value of $5.3 \mathrm{~dB}$ is obtained. As the focusing effect is attributed to phase delay introduced by diffraction along the wave front, the pupil mask centred on the face encountering the plane wave increases the diffraction inside the cuboid. This one causes a greater difference between the phase velocities between the surrounding medium and inside the cuboid, as seen in Figure 2c-2d.

The size of the pupil mask is an important factor in the enhancement since, if its size is greater, the intensity that penetrates the cuboid will be smaller. Figure 3a shows the longitudinal relative sound intensity distributions of a cuboid lens without a pupil-mask and, as an example, masked with three different mask side dimensions: $30 \mathrm{~mm}(0.375 \lambda), 15 \mathrm{~mm}(0.1875 \lambda)$ and $5 \mathrm{~mm}$ $(0.0625 \lambda)$. It has been observed that there are two intensity peaks, one inside and the other outside the cuboid lens. The external peak amplitude increases as the mask size is reduced and, at the same time, its focus position moves closer to the cuboid shadow surface. The internal peak dominates over the outside peak when the mask side dimension is $30 \mathrm{~mm}$. In this case, there is no intensity enhancement, on the contrary, the intensity of the outside peak is reduced. In the case of a cubic lens without a pupil mask, the wave front inside the cube is formed due to the interaction of a plane incident wave with the faces of the cube and the gas-air boundary. 
In the case of an amplitude mask on the illuminated side of the cube, the wave front inside it is formed as additional toroidal fronts, the transverse dimension of which is determined by the distance between the outer boundaries of the mask and the cube. In the case of a relatively large pupil mask size, the wave front converges to the axis inside the cube at a wide angle, which leads to an increase in the intensity of the maximum inside the cube. Figure $3 \mathrm{a}$ shows the effect of pupil mask size on forming a diffraction limited focus of a lens. With a small mask size, the wave front convergence area moves to the shadow surface of the cube, which reduces the intensity of the maximum inside the cube and increases the intensity in the focusing area. Figure 4 shows the longitudinal relative sound intensity distributions for the square pupil-mask alone. This is, without the cuboid, where the enhancement caused by diffraction is evaluated.

Formation of the focusing area near the shadow surface of the cuboid lens is due to the formation of positive curvature of sound velocity near the shadow surface (the wave front inside the cube becomes concave near the shadow side of the lens, instead of being convex near the illuminated side of the lens). This is because of the difference in velocities at the centre and near the edge of the lens. This corresponds to the focusing conditions [32]. The enhancement produced by the cubic lens with a pupil mask is due to a coupled action between the cubic lens and the mask. The focusing effect is clearly visible for a pupil of $30 \mathrm{~mm}$ in size the near axial distance $\mathrm{z}=0.7 \lambda$.

On the other hand, it has been observed that the pupil-mask, in addition to an enhancement in the focusing produces a resolution improvement, which constitutes a reduction in FWHM. Figure $3 \mathrm{~b}$ shows the transverse relative sound intensity distributions in the focus of a cubic particle-lens. This fact can be observed more clearly in Figure 5 where the resolution, $r$, as a function of the mask size is presented. It has been observed that, as the size of the mask is reduced, there is a resolution improvement. With the $5 \mathrm{~mm}(0.0626 \lambda)$ side pupil-mask, an 
improvement in the resolution of around $15 \%$ is obtained, which gets close to the diffraction limit.

\section{Conclusions}

In conclusion, it has been shown that intensity loss is not unavoidable in the acoustical cuboid gas-filled lens applied by apodization, and the anomaly apodization effect is valid for this type of acoustical lens, which was not obvious. The sound intensity enhancement produced by a pupil-mask in $1-\lambda$ dimension gas-filled cuboid lens, was shown in simulation. This is the smallest size of an acoustic lens ever considered in the literature of this field. Results show that it is possible to simultaneously obtain a sound intensity enhancement and a resolution improvement compared to the same sized non-masked model by the addition of a pupil-mask located on the plane wave incident face. Although increments of $10-15 \%$ are not large, they were observed for a new type of acoustic lens with a $1-\lambda$ dimension. This simple mesoscale gas-filled lens, due to its flat design and a geometry matching with most of the acoustic sources, could be the motivation for several applications in science and technology ranging from aeroacoustics to ultrasound therapy (with a corresponding selection of acoustic impedances and a speed of sound contrast medium in a lens). These results could open new possibilities in the design of small, lightweight low cost acoustic lenses with a low contrast of impedances with the host medium and without the need of using metamaterials or exotic gases.

\section{Acknowledgements}

This work has been supported by TEC2015-70939-R (MINECO/FEDER). The research was partially supported by Tomsk Polytechnic University Competitiveness Enhancement Program.

\section{References}


[1] E. Atkinson,.Elementary treatise on physics, experimental and applied(W. Wood and Co, $11^{\text {th }}$ edition, New York, USA, 1882), pp. 237.

[2] J.M. Kendall, NASA Tech Briefs 5(3), (1980), 345-46.

[3] D.C. Thomas, K. L.Gee, R.S. Turley, Am. J. Phys. 77(3), (2009), 197-203.

[4] D.C. Calvo, A.L. Thangawng, M. Nicholas, and C.N. Layman, Appl. Phys. Lett., 107, (2015), 014013.

[5] F. Cervera; J. V. Sanchez-Perez; R. Martinez-Sala R, C. Rubio; F. Meseguer; C. Lopez, D. Caballero and J. Sánchez-Dehesa. Phys.Rev.Lett., 88(2), (2002), 2390223906,

[6] A.Sukhovich, B.Merheb, K.Muralidharan, J.O.Vasseur, Y.Pennec, P.A.Deymier, J.H.Page, Phys.Rev.Lett., 102, (2009), 154301,.

[7] P. Peng, B. Xiao, Y. Wu, Phys. Lett. A, 378, (2014), 3389.

[8] K. Tang, C. Y. Qiu, M. Z. Ke, J. Y. Lu, Y. T. Ye, Z. Y. Liu, Sci. Rep., 4, (2014), 6517.

[9] Y. Li, B. Liang, X. Tao, X. F. Zhu, X. Y.Zou, J. C. Cheng, Appl. Phys. Lett., 101, (2012), 233508.

[10] Y. Li, B. Liang, Z. M. Gu, X. Y. Zou, J. C. Cheng, Sci. Rep. 3, (2013), 2546.

[11] M. Moleron, M. Serra-Garcia, C. Daraio, Appl. Phys. Lett., 105, (2014), 114109.

[12] Z. Lin, X. Guo, J. Tu, J. Cheng, J. Wu, D.Zhang, J. of Appl. Phys., 115, (2014), 104504.

[13] R.A.Jahdali and Y.Wu. Appl. Phys. Lett. 108, (2016), 031902.

[14] X.D. Fan, Y. F. Zhu, B. Liang, J. Yang, L. L. Yin, J. Yang, J. C. Cheng, Appl. Phys. Lett. 109, (2016), 153501.

[15] X. D. Fan, Y. F. Zhu, B. Liang, J. Yang, J. C. Cheng, Appl. Phys. Lett. 109, (2016), 243501.

[16] B.S. Lukiyanchuk, R. Paniagua-Domínguez, I.V. Minin, O.V. Minin, W. Zengbo, Opt. Mater. Express, 7(6), (2017), 1820-1847.

[17] I.V. Minin, O. V. Minin, I. S. Nefedov, Opt. Lett., 41(3), (2016), 785. 
[18] H. Wang, Colin J.R. Sheppard, K. Ravi, S.T. Ho, and G. Vienne. Laser Photonics Rev., (2011), 1-39.

[19] D. R. Dietz, C. G.Oakley, D. Morgan, D. Patwa, United States Patent. (2011), Patent No.: US 7,888,847 B2

[20] V.L.S. Nantes-Button, E. Tavares-Costa, J. M. Maia, R. G. Dantas, Proc. SPIE 3982, Medical Imaging 2000: Ultrasonic Imaging and Signal Processing, (2000) 132-141.

[21] I. V. Minin, O. V. Minin, Patent of Russia N, 21, (2015), 153686.

[22] B. Yan, L. Yue, Z. B. Wang, Opt. Commun., 370, (2016), 140.

[23] L. Yue, B. Yan, J. N. Monks, Z. B. Wang, N. T. Tung, V. D. Lam, O. V. Minin, I. V. Minin, J. Phys. D: Appl. Phys., 50, (2017), 175102.

[24] L. Yue, B. Yan, J. N. Monks, R. Dhama, Z. Wang, O. V. Minin, I. V. Minin, Ann. Phys.., 530, (2018), 1700384

[25] L. Yue, B. Yan, J. N. Monks, R. Dhama, Z. Wang, O. V. Minin, I. V. Minin, J. Infrared. Milli. Terahz. Waves, 39, (2018), 546-552.

[26] O.V. Minin, I.V. Minin, Opt. Quant. Electron, 49, (2017), 49-54.

[27] J.H.Lopes, J.P.Leão-Neto, I.V.Minin, O.V.Minin, G.T.Silva, in Proceedings of the 22nd International Congress on Acoustics, ICA2016, Buenos Aires, Argentina, (2016).

[28] J.H. Lopes, M.A.B Andrade, J.P. Leao-Neto, J.C. Adamowski, I.V. Minin, G.T. Silva, Phys. Rev. Appl., 8, (2017), 024013.

[29] I.V.Minin and O.V.Minin. MATEC Web of Conferences, 155 (2018); DOI $10.1051 /$ matecconf/201815501029

[30] C. Rubio, D. Tarrazo-Serrano, O.V. Minin, A. Uris, I.V. Minin, Europhys. Lett, accepted (2018).

[31] J. Berenguer, J. Compt. Physics, 114, (1994), 185.

[32] B.G. Lucas and T.G. Muir, J. Acoust. Soc. Am. 72, (1982) 1289. 


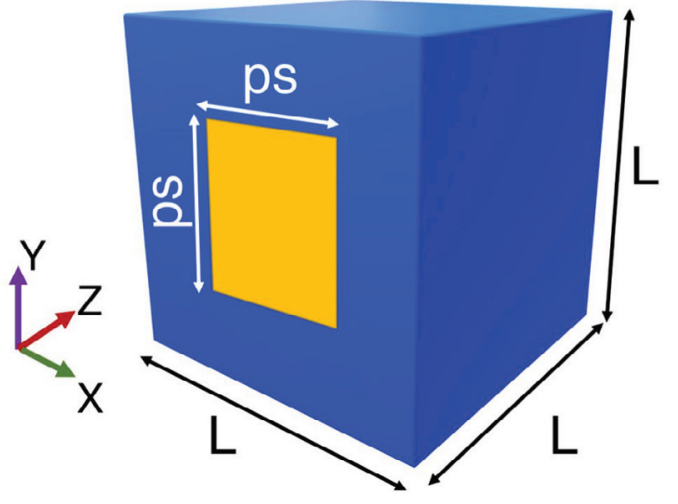

Figure 1. Schematic diagram of the cuboid lens with pupil mask. 

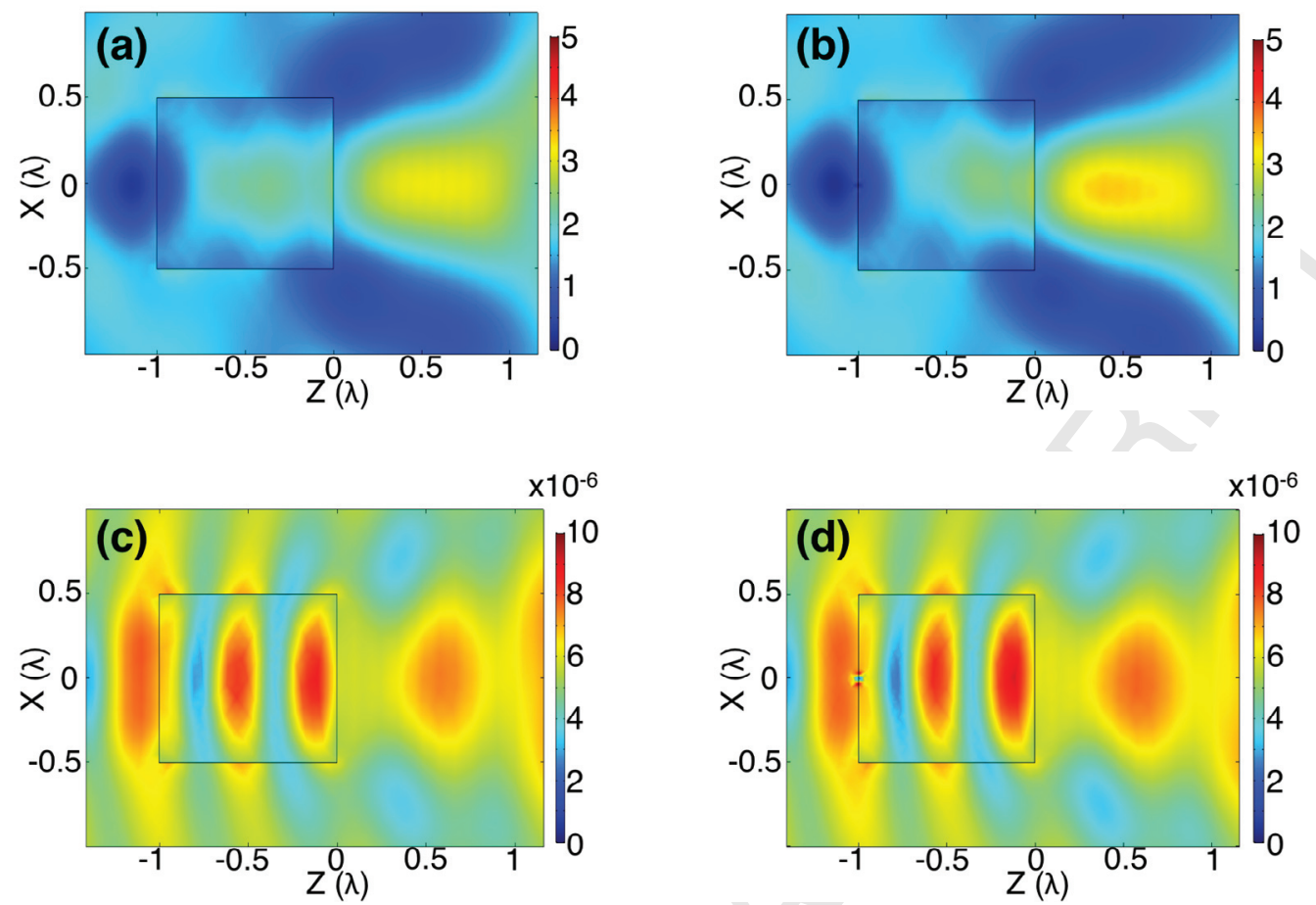

Figure 2. Normalized sound intensity, I/Iincident, distributions for $\mathrm{CO}_{2}$ filled cuboid lens (a) without pupil mask and (b) with square pupil-mask of $5 \mathrm{~mm}$ side $(0.0625 \lambda)$. Normalized sound velocity, $U_{\text {Air }} / U_{R M S}$, maps for cuboid lens (c) without pupil mask and (d) with square pupilmask of $5 \mathrm{~mm}$ side $(0.0625 \lambda)$ 

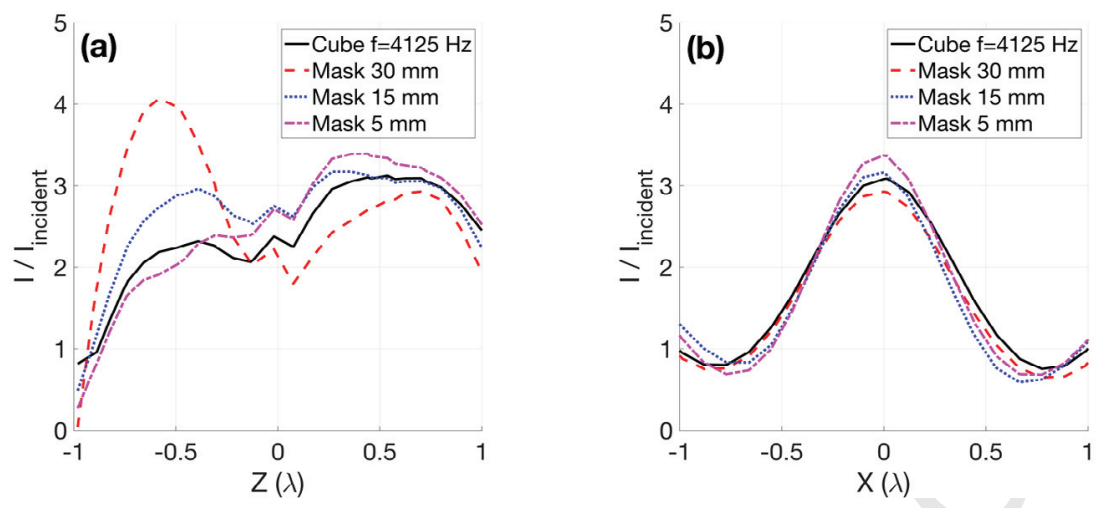

Figure 3. (a) Longitudinal relative sound intensity distributions for the cuboid lens without pupil mask and square pupil-mask of 30,15 and $5 \mathrm{~mm}$ side. (b) Longitudinal relative sound intensity distributions in the focus for the cuboid lens without pupil mask and with a square pupil-mask of 30, 15 and $5 \mathrm{~mm}$ side. 


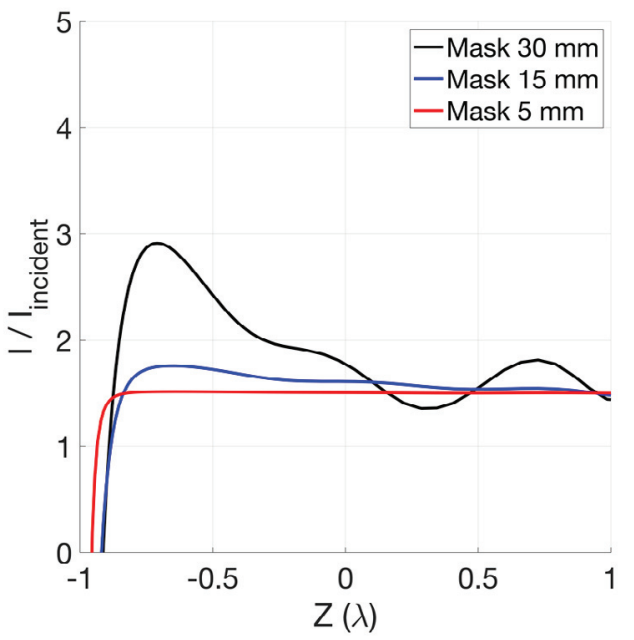

Figure 4. Longitudinal relative sound intensity distributions for the square pupil-mask alone (without the cuboid) of 30,15 and $5 \mathrm{~mm}$ side. 


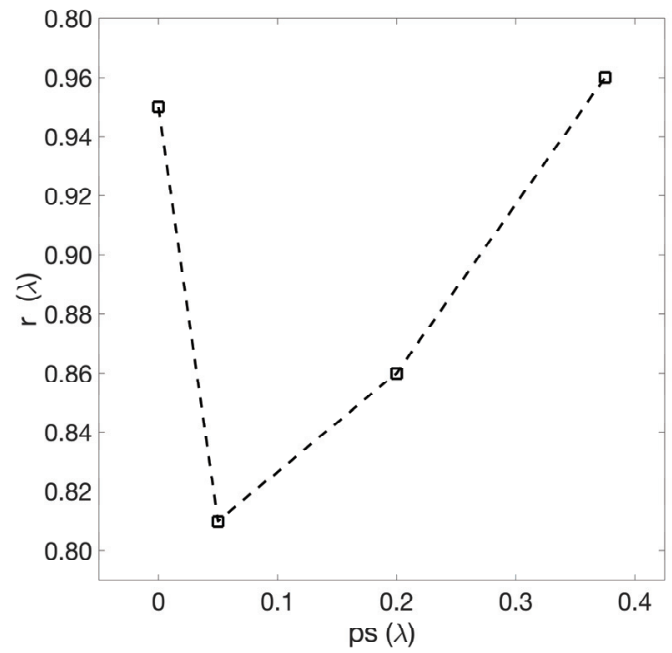

Figure 5. Resolution, r, for the cuboid lens with and without pupil mask. 